\title{
On the choice of parameters for a model of small window
}

\author{
A. S. Melikhova, M. P. Faleeva, I. Y. Popov \\ ITMO University, Kroverkskiy pr. 49, St. Petersburg, 197101, Russia \\ alina.s.melikhova@gmail.com, faleeva.masha@gmail.com, popov1955@gmail.com
}

PACS 73.23.-b, 73.23.Hk, 73.23.Ra, 03.65.Yz

DOI 10.17586/2220-8054-2021-12-2-151-155

Scattering of plane waves and Gaussian beams by a screen with small opening is considered. The asymptotics far from the obstacle is obtained. The results can be useful for description of perforated nanolayers and for fitting the model based on the operator extensions theory.

Keywords: scattering, Green's function, asymptotics.

Received: 25 March 2021

\section{Introduction}

Wave scattering by perforated screens was studied over a long period [1]. Nevertheless, last decade, one observe an intensive discussion of the problem related to nanoscience, e.g., to studying of perforated nanolayers and nanofilms (see, e.g., [2,3]). Several mathematical models were suggested for the process description, particularly, a model of point-like windows based the theory of self-adjoint extensions of symmetric operators [4-6]. The model is explicitly solvable. It is an evident advantage. Unfortunately, fitting of the model is not simple. One should consider the asymptotics of the solution for the realistic problem if the window is small (see, e.g., [7-9]) and choose the proper values of the model parameters ensuring good approximation of the realistic solution by the model one. For this purpose, it is possible to consider a benchmark problem with simpler geometry [10]. In our case, we can deal with a plane with an orifice. In the present paper we consider the scattering problem for the plane $z=0$ in $\mathbb{R}^{3}$ with the Cartesian coordinates $(x, y, z)$.

\section{Scattering}

Let the incoming plane wave is in the upper half-space $z>0$. Correspondingly, the reflected wave is also in this half-space. Consider the scattered wave $U$ in the lower half-space. Function $U(\mathbf{r})$ (where $\mathbf{r}=\{r, \theta, \varphi\}$ in spherical coordinates or $\mathbf{r}=\{x, y, z\}$ in Cartesian coordinates) can be written in the following form:

$$
U(\mathbf{r})=\iint_{D} \frac{\partial}{\partial \mathbf{n}^{\prime}} G\left(\mathbf{r}, \mathbf{r}^{\prime}, k\right) \psi_{i n}\left(\mathbf{r}^{\prime}\right) d \mathbf{r}^{\prime},
$$

where $D$ is a window (aperture), $\psi_{i n}$ is the incoming wave, $G$ is the Green function, $k$ is the wave number. Here vector $\mathbf{r}^{\prime}$ can be also presented in spherical or Cartesian coordinates with following notations: $\mathbf{r}^{\prime}=\left\{r^{\prime}, \theta^{\prime}, \varphi^{\prime}\right\}$ (in spherical coordinates), $\mathbf{r}^{\prime}=\left\{x^{\prime}, y^{\prime}, z^{\prime}\right\}$ (in Cartesian coordinates).

Let one examine integrand functions from (1) separately. Firstly, consider the normal derivative of Green's function. It can be presented as follows:

$$
\frac{\partial}{\partial \mathbf{n}^{\prime}} G\left(\mathbf{r}, \mathbf{r}^{\prime}, k\right)=\frac{1}{4 \pi} \frac{\partial}{\partial \mathbf{n}^{\prime}}\left(\frac{\mathrm{e}^{\mathrm{i} k\left|\mathbf{r}-\mathbf{r}^{\prime}\right|}}{\left|\mathbf{r}-\mathbf{r}^{\prime}\right|}-\frac{\mathrm{e}^{\mathrm{i} k\left|\mathbf{r}-\mathbf{r}^{\prime \star}\right|}}{\left|\mathbf{r}-\mathbf{r}^{\prime \star}\right|}\right) .
$$

It is clear that in our case the normal derivative $\partial / \partial \mathbf{n}^{\prime}$ is equal to the derivative with respect to the $Z^{\prime}$-axis (in Cartesian coordinates).

One can obtain:

$$
\frac{\partial}{\partial \mathbf{n}^{\prime}} G\left(\mathbf{r}, \mathbf{r}^{\prime}, k\right)=-\frac{z}{2 \pi} \frac{\mathrm{e}^{\mathrm{i} k\left|\mathbf{r}-\mathbf{r}^{\prime}\right|}}{\left|\mathbf{r}-\mathbf{r}^{\prime}\right|}\left(\frac{\mathrm{i} k}{\left|\mathbf{r}-\mathbf{r}^{\prime}\right|}-\frac{1}{\left|\mathbf{r}-\mathbf{r}^{\prime}\right|^{2}}\right) .
$$

Now we can examine the second integrand function $\psi_{i n}\left(\mathbf{r}^{\prime}\right)$. In upper half-plane it can presented as a following sum:

$$
\psi_{i n}\left(\mathbf{r}^{\prime}\right)=\mathrm{e}^{\mathrm{i} \mathbf{k}^{i n} \cdot \mathbf{r}^{\prime}}+\mathrm{e}^{\mathrm{i} \mathbf{k}^{r e f} \cdot \mathbf{r}^{\prime}}
$$

where vectors $\mathbf{k}^{i n}$, $\mathbf{k}^{r e f}$ and $\mathbf{r}^{\prime}$ can be described as follows:

$$
\begin{aligned}
& \mathbf{k}^{i n}=k \mathbf{v}^{i n}, \quad \mathbf{k}^{r e f}=k \mathbf{v}^{r e f}, \quad \mathbf{r}^{\prime}=r^{\prime} \mathbf{v}^{\prime} ; \quad\left|\mathbf{v}^{i n}\right|=\left|\mathbf{v}^{r e f}\right|=\left|\mathbf{v}^{\prime}\right|=1 ; \\
& \mathbf{v}^{i n}=\left\{1 ; \varphi_{\text {in }} ; \theta_{\text {in }}\right\}, \quad \mathbf{v}^{r e f}=\left\{1 ; \varphi_{\text {ref }} ; \theta_{\text {ref }}\right\}=\left\{1 ; \varphi_{\text {in }} ; \pi-\theta_{i n}\right\}, \quad \mathbf{v}^{\prime}=\left\{1 ; \varphi^{\prime} ; \theta^{\prime}\right\} .
\end{aligned}
$$


Let us consider the solution in the lower half-plane where the reflected plane wave is absent:

$$
\psi_{\text {in }}\left(\mathbf{r}^{\prime}\right)=\mathrm{e}^{\mathrm{i} \mathbf{k}^{i n} \cdot \mathbf{r}^{\prime}}=\mathrm{e}^{\mathrm{i} k r^{\prime} \mathbf{v}^{i n} \cdot \mathbf{v}^{\prime}},
$$

Since we are mostly interested in asymptotic behavior of function $U(\mathbf{r})$ when $r$ tends to infinity, we can express function $U(\mathbf{r})$ from (1) in the following asymptotic form:

$$
U(r, \theta, \varphi) \approx A(\theta, \varphi) \frac{\mathrm{i} k \mathrm{e}^{\mathrm{i} k r}}{r} \text { for } r \rightarrow \infty,
$$

and then examine only the coefficient $A(\theta, \varphi)$. To meet this goal we have to single out the factor $\mathrm{i} k \mathrm{e}^{\mathrm{i} k r} / r$ from the formula (1).

First of all, we can properly approximate expression for the normal derivative of Green's function (3). We will do it in several steps. In the first step, we can approximate the expression for the modulus of the difference of radius vectors $\mathbf{r}$ and $\mathbf{r}^{\prime}$ :

$$
\begin{aligned}
\left|\mathbf{r}-\mathbf{r}^{\prime}\right|=\sqrt{r^{2}+\left(r^{\prime}\right)^{2}-2 r r^{\prime} \cos \left(\hat{\left.\mathbf{r}, \mathbf{r}^{\prime}\right)}\right.}=r \sqrt{1+\frac{\left(r^{\prime}\right)^{2}}{r^{2}}-2 \frac{r^{\prime}}{r} \cos \left(\hat{\left.\mathbf{r}, \mathbf{r}^{\prime}\right)}\right.} & \approx \\
& \approx r-r^{\prime} \cos \left(\mathbf{r}, \mathbf{r}^{\prime}\right) \text { for } r \rightarrow \infty
\end{aligned}
$$

In the second step, one can find an approximation for the second factor in the expression (3) as follows:

$$
\begin{aligned}
\frac{\mathrm{e}^{\mathrm{i} k\left|\mathbf{r}-\mathbf{r}^{\prime}\right|}}{\left|\mathbf{r}-\mathbf{r}^{\prime}\right|} \approx \frac{\mathrm{e}^{\mathrm{i} k r} \mathrm{e}^{-\mathrm{i} k r^{\prime} \cos \left(\mathbf{r}, \hat{\mathbf{r}}^{\prime}\right)}}{r\left(1+\frac{1}{2}\left(\frac{\left(r^{\prime}\right)^{2}}{r^{2}}-2 \frac{r^{\prime}}{r} \cos \left(\hat{\mathbf{r}}, \mathbf{r}^{\prime}\right)\right)\right)} & \\
\approx \frac{\mathrm{e}^{\mathrm{i} k r}}{r} \mathrm{e}^{-\mathrm{i} k r^{\prime} \cos \left(\mathbf{r}, \hat{\mathbf{r}}^{\prime}\right)}(1- & \left.\frac{1}{2}\left(\frac{\left(r^{\prime}\right)^{2}}{r^{2}}-2 \frac{r^{\prime}}{r} \cos \left(\mathbf{r}, \mathbf{r}^{\prime}\right)\right)\right) \approx \\
& \approx \frac{\mathrm{e}^{\mathrm{i} k r}}{r} \mathrm{e}^{-\mathrm{i} k r^{\prime} \cos \left(\mathbf{r}, \hat{\mathbf{r}}^{\prime}\right)}\left(1+\frac{r^{\prime}}{r} \cos \left(\mathbf{r}, \hat{\mathbf{r}^{\prime}}\right)\right) \text { for } r \rightarrow \infty .
\end{aligned}
$$

In the next step, one can easily find approximations for the terms in the third factor in the expression (3):

$$
\begin{aligned}
& \frac{\mathrm{i} k}{\left|\mathbf{r}-\mathbf{r}^{\prime}\right|} \approx \frac{\mathrm{i} k}{r}\left(1+\frac{r^{\prime}}{r} \cos \left(\mathbf{r}, \hat{\mathbf{r}}^{\prime}\right)\right) \text { for } r \rightarrow \infty ; \\
& \frac{1}{\left|\mathbf{r}-\mathbf{r}^{\prime}\right|^{2}}=\frac{1}{r^{2}\left(1+\frac{\left(r^{\prime}\right)^{2}}{r^{2}}-2 \frac{r^{\prime}}{r} \cos \left(\hat{\left.\left.\mathbf{r}, \mathbf{r}^{\prime}\right)\right)}\right.\right.} \approx \frac{1}{r^{2}} \text { for } r \rightarrow \infty .
\end{aligned}
$$

And finally, by substituting expressions (5)-(8) into formula (3) we derive an approximate expression for the normal derivative of Green's function in the following form:

$$
\frac{\partial}{\partial \mathbf{n}^{\prime}} G\left(\mathbf{r}, \mathbf{r}^{\prime}, k\right)=-\frac{z}{2 \pi} \frac{\mathrm{e}^{\mathrm{i} k\left|\mathbf{r}-\mathbf{r}^{\prime}\right|}}{\left|\mathbf{r}-\mathbf{r}^{\prime}\right|}\left(\frac{\mathrm{i} k}{\left|\mathbf{r}-\mathbf{r}^{\prime}\right|}-\frac{1}{\left|\mathbf{r}-\mathbf{r}^{\prime}\right|^{2}}\right) \approx-\frac{\cos \theta}{2 \pi} \frac{\mathrm{i} k \mathrm{e}^{\mathrm{i} k r}}{r} \mathrm{e}^{-\mathrm{i} k r^{\prime} \cos \left(\mathbf{r}, \hat{\mathbf{r}}^{\prime}\right)} \text { for } r \rightarrow \infty .
$$

Secondly, by matching expressions (1), (9) with asymptotic formula (4), one can obtain the following form of the coefficient $A(\theta, \varphi)$ :

$$
A(\theta, \varphi)=-\frac{\cos \theta}{2 \pi} \iint_{D} \mathrm{e}^{-\mathrm{i} k r^{\prime} \cos \left(\mathbf{r}, \hat{\mathbf{r}}^{\prime}\right)} \mathrm{e}^{\mathrm{i} k r^{\prime} \mathbf{v}^{i n} \cdot \mathbf{v}^{\prime}} d \mathbf{r}^{\prime} .
$$

And due to the last formula one can examine the asymptotic behaviour of the function $U(\mathbf{r})$ for every specific domain $D$.

\section{A round domain $D$}

In this section we will discuss the case when the domain $D$ is a circle in the plane $z=0$ of radius $R$ :

$$
D=\left\{\left(x^{\prime}, y^{\prime}\right):\left(x^{\prime}\right)^{2}+\left(y^{\prime}\right)^{2} \leq R^{2}\right\} \text { or } D=\left\{\left(r^{\prime}, \varphi^{\prime}\right): 0 \leq r^{\prime} \leq R^{2}, \varphi^{\prime} \in[0 ; 2 \pi)\right\}
$$

For such domain $D$ we can find the coefficient $A(\theta, \varphi)$ in non-integral form. To do this we should investigate the integrand function from (10) at first and after few simplifications integrate it. 
Let examine function $\mathrm{e}^{-\mathrm{i} k r^{\prime} \cos \left(\mathbf{r}, \hat{\mathbf{r}}^{\prime}\right)} \mathrm{e}^{\mathrm{i} k r^{\prime} \mathbf{v}^{i n} \cdot \mathbf{v}^{\prime}}$. Firstly, we can present $\cos \left(\hat{\mathbf{r}}, \mathbf{r}^{\prime}\right)$ as a function of angles $\theta, \varphi$ and $\varphi^{\prime}:$

$$
\cos \left(\hat{\mathbf{r}, \mathbf{r}^{\prime}}\right)=\frac{\mathbf{r} \cdot \mathbf{r}^{\prime}}{r r^{\prime}}=\left(\cos \varphi \cos \varphi^{\prime}+\sin \varphi \sin \varphi^{\prime}\right) \sin \theta=\cos \left(\varphi-\varphi^{\prime}\right) \sin \theta
$$

Secondly, we can examine scalar products from $\mathrm{e}^{\mathrm{i} k r^{\prime} \mathbf{v}^{i n} \cdot \mathbf{v}^{\prime}}$ :

$$
\begin{aligned}
\mathbf{v}^{i n} \cdot \mathbf{v}^{\prime}=\cos \varphi_{i n} \sin \theta_{i n} \cos \varphi^{\prime} \sin \theta^{\prime}+ & \sin \varphi_{i n} \sin \theta_{i n} \sin \varphi^{\prime} \sin \theta^{\prime}+\cos \theta_{i n} \cos \theta^{\prime}= \\
& =\left(\cos \varphi_{i n} \cos \varphi^{\prime}+\sin \varphi_{i n} \sin \varphi^{\prime}\right) \sin \theta_{i n}=\cos \left(\varphi_{i n}-\varphi^{\prime}\right) \sin \theta_{i n}
\end{aligned}
$$

From (11), it follows:

$$
\mathrm{e}^{\mathrm{i} k r^{\prime} \mathbf{v}^{i n} \cdot \mathbf{v}^{\prime}}=\mathrm{e}^{\mathrm{i} k r^{\prime} \cos \left(\varphi_{i n}-\varphi^{\prime}\right) \sin \theta_{i n}} .
$$

And finally, (3) and (12) yield us the following result about the integrand function being under discussion:

$$
\mathrm{e}^{-\mathrm{i} k r^{\prime} \cos \left(\mathbf{r}, \hat{\mathbf{r}}^{\prime}\right)} \mathrm{e}^{\mathrm{i} k r^{\prime} \mathbf{v}^{i n} \cdot \mathbf{v}^{\prime}}=\mathrm{e}^{\mathrm{i} k r^{\prime}\left(\cos \left(\varphi_{i n}-\varphi^{\prime}\right) \sin \theta_{i n}-\cos \left(\varphi-\varphi^{\prime}\right) \sin \theta\right)}
$$

It remains only to integrate expression (10) using the form (13) of integrand function:

$$
\begin{aligned}
& A(\theta, \varphi)=-\frac{\cos \theta}{2 \pi} \iint_{D} \mathrm{e}^{-\mathrm{i} k r^{\prime} \cos \left(\hat{\mathbf{r}}, \mathbf{r}^{\prime}\right)} \mathrm{e}^{\mathrm{i} k r^{\prime} \mathbf{v}^{i n} \cdot \mathbf{v}^{\prime}} d \mathbf{r}^{\prime}= \\
& \quad=-\frac{\cos \theta}{2 \pi} \int_{0}^{R} r^{\prime}\left(\int_{0}^{2 \pi} \exp \left(\mathrm{i} k r^{\prime}\left(\cos \left(\varphi_{i n}-\varphi^{\prime}\right) \sin \theta_{i n}-\cos \left(\varphi-\varphi^{\prime}\right) \sin \theta\right)\right) d \varphi^{\prime}\right) d r^{\prime} .
\end{aligned}
$$

Firstly, one can calculate the inner integral from (14):

$$
\begin{aligned}
& \int_{0}^{2 \pi} \exp \left(\mathrm{i} k r^{\prime}\left(\cos \left(\varphi_{i n}-\varphi^{\prime}\right) \sin \theta_{i n}-\cos \left(\varphi-\varphi^{\prime}\right) \sin \theta\right)\right) d \varphi^{\prime}= \\
& \int_{0}^{2 \pi} \exp \left(\mathrm{i} k r^{\prime}\left(c_{1} \cos \varphi^{\prime}+c_{2} \sin \varphi^{\prime}\right)\right) d \varphi^{\prime}=2 \pi I_{0}\left(\sqrt{-\left(k r^{\prime} c_{1}\right)^{2}-\left(k r^{\prime} c_{2}\right)^{2}}\right)= \\
& =2 \pi I_{0}\left(\mathrm{i} k \sqrt{c_{1}^{2}+c_{2}^{2}} r^{\prime}\right)=2 \pi I_{0}\left(\mathrm{i} k \Theta\left(\theta_{i n}, \varphi_{i n} ; \theta, \varphi\right) r^{\prime}\right) \\
& \Theta\left(\theta_{i n}, \varphi_{i n} ; \theta, \varphi\right) \stackrel{d e f}{=} \sqrt{\sin ^{2} \theta_{i n}-2 \cos \left(\varphi_{i n}-\varphi\right) \sin \theta_{i n} \sin \theta+\sin ^{2} \theta}
\end{aligned}
$$

where $I_{0}(z)$ is the modified Bessel function of the first kind of the 0-th order. Secondly, one can calculate the outer integral from (17) excluding constant terms:

$$
\int_{0}^{R} r^{\prime} I_{0}\left(\mathrm{i} k \Theta\left(\theta_{i n}, \varphi_{i n} ; \theta, \varphi\right) r^{\prime}\right) d r^{\prime}=\frac{R J_{1}\left(k \Theta\left(\theta_{i n}, \varphi_{i n} ; \theta, \varphi\right) R\right)}{k \Theta\left(\theta_{i n}, \varphi_{i n} ; \theta, \varphi\right)}
$$

where $J_{1}(z)$ is the Bessel function of the first kind of the 1-st order. Thus, one can obtain coefficient $A(\theta, \varphi)$ as follows:

$$
A(\theta, \varphi)=-\frac{\cos \theta}{\pi} \pi \frac{R J_{1}\left(k \Theta\left(\theta_{i n}, \varphi_{i n} ; \theta, \varphi\right) R\right)}{k \Theta\left(\theta_{i n}, \varphi_{i n} ; \theta, \varphi\right)}=-\frac{R \cos \theta}{\Theta\left(\theta_{i n}, \varphi_{i n} ; \theta, \varphi\right)} \frac{J_{1}\left(k \Theta\left(\theta_{i n}, \varphi_{i n} ; \theta, \varphi\right) R\right)}{k} .
$$

For further discussions we can rewrite the obtained expression in more convenient for us form:

$$
A(\theta, \varphi)=-R^{2} \cos \theta \frac{J_{1}\left(\Theta\left(\theta_{i n}, \varphi_{i n} ; \theta, \varphi\right) k R\right)}{\Theta\left(\theta_{i n}, \varphi_{i n} ; \theta, \varphi\right) k R} .
$$




\section{A round domain $D$ : discussions.}

First of all, one can note that in the case of normal wave incident function $\Theta\left(\theta_{i n}, \varphi_{i n} ; \theta, \varphi\right)$ is very simple and does not depend on $\varphi_{\text {in }}$ and $\varphi$ :

$$
\Theta\left(\theta_{i n}, \varphi_{i n} ; \theta, \varphi\right)=\left|\theta_{i n}=\pi\right|=\Theta_{\text {norm }}(\theta)=|\sin \theta| .
$$

Secondly, in the case with $k R \ll 1$ one can easily obtain the following approximation:

$$
A(\theta, \varphi) \approx-R^{2} \cos \theta \frac{\Theta\left(\theta_{i n}, \varphi_{i n} ; \theta, \varphi\right) k R / 2}{\Theta\left(\theta_{i n}, \varphi_{i n} ; \theta, \varphi\right) k R}=-\frac{R^{2}}{2} \cos \theta \text { for } k R \ll 1 .
$$

Id est, in this case coefficient $A(\theta, \varphi)$ does not depend on $\varphi$ and is proportional to $\cos \theta$. In other cases we have a non-trivial dependence of this coefficient on $\varphi$.

As for the model of point-like window in the screen, it looks like the following one. Let us consider the Laplace operators in $L_{2}\left(\Omega^{+}\right)$and $L_{2}\left(\Omega^{-}\right)$with Neumann boundary conditions on $\partial \Omega^{ \pm}$(here $\Omega^{ \pm}$is upper (lower) half-plane), namely

$$
\left.\frac{\partial f}{\partial n}\right|_{\partial \Omega^{ \pm}}=0,
$$

where $n$ denotes the external unit normal vector to $\partial \Omega^{ \pm}$, i.e. $n=(0,0, \pm 1)$.

Let us construct a point-like window at point $x^{0} \in \partial \Omega^{ \pm}$. If we restrict the considered operators onto the set of smooth functions that vanish in the neighbourhood of $x^{0}$, it is well known that the closures $\Delta_{0}^{+}$and $\Delta_{0}^{-}$of the considered operators are symmetric operators with deficiency indices $(1,1)$. Thus, the operator

$$
\Delta_{0}=\Delta_{0}^{+} \oplus \Delta_{0}^{-}
$$

is symmetric and has deficiency indices $(2,2)$. As long as the deficiency indices are equal, we may conclude that the considered operator does have self-adjoint extensions. This operator gives one the model in question.

However, if we impose the Dirichlet boundary conditions

$$
\left.u\right|_{\partial \Omega^{ \pm}}=0,
$$

we immediately face the following problem: the operator obtained via restriction onto the set of smooth functions that vanish in the vicinity of $x^{0} \in \partial \Omega$ is essentially self-adjoint. There is no element in the $L_{2}$ space which can be taken as a deficiency element. All candidates have too strong singularities. Respectively, constructing of the model is based on the extension of the initial space up to a so-called Pontryagin space (with indefinite inner product). The version of the model for the Dirichlet case based on the theory of self-adjoint operator extensions in Pontryagin space was suggested in $[6,11,12]$. It is important to choose the extension parameters in such a way that the model solution coincides with the main term of the asymptotics (in the window width) of the realistic solution, corresponding to small windows $[5,6]$. It can be made by comparison of the asymptotics of the scattering problem described above and the model solution ( [6]).

One more application of the result is atmospheric quantum communication description. For quantum channel through turbulent atmosphere, the main reason for error is a shift in the Gaussian beam with respect to the input aperture of the receiver $[13,14]$. For calculation of this error, one can use the obtained formulas with the replacement of plane waves by the Gaussian beam [15], e.g., such as:

$$
\psi=\frac{w_{0}}{w} \exp \left(-i k(L-z)+i \phi-\left(\frac{i k}{2 b}+\frac{1}{w^{2}}\right)\left(x^{2}+y^{2}\right)\right)
$$

where $w, w_{0}, \phi, b$ are parameters of the Gaussian beam, $L$ is the distance from the sender, $x, y, z$ are the Cartesian coordinates. One can also take higher modes of the Gaussian beam.

\section{Aknowledgements}

This work was partially financially supported by grant 19-31-90154 of RFBR.

\section{References}

[1] Morse F.M., Feshbach G. Methods of theoretical physics, V. 2. Foreign Literature Publishing House, Moscow, 1960,986 p.

[2] H. Wang, Sh. Zou. Extremely low scattering cross section of a perforated silver film. Appl. Phys. Lett., 2009, 94, P. 073119.

[3] Mirin N.A., Ali T.A., Nordlander P., Halas N.J. Perforated semishells: far-field directional control and optical frequency magnetic response. ACS Nano, 2010, 4(5), P. 2701-2712.

[4] Kiselev A.A., Pavlov B.S. The eigenfrequencies and eigenfunctions of the Laplace operator of the Neumann problem in a system of two coupled resonators. Theor. Math. Phys., 1994, 100, P. 354-366.

[5] Popov I.Yu. The extension theory and localization of resonances for the domain of trap type. Mathematics of the USSR-Sbornik, 1992, 71(1), P. 209-234. 
[6] Popov I.Yu. The resonator with narrow slit and the model based on the operator extensions theory. J. Math. Phys., 1992, 33(11), P. 3794-3801.

[7] Gadyl'shin R.R. Influence of the position of the opening and its shape on the properties of a Helmholtz resonator. Theor. Math. Phys., 1992, 93, P. 1151-1159.

[8] Borisov D., Exner P. Distant perturbation asymptotics in window-coupled waveguides. I. The non-threshold case. J. Math. Phys., 2006, 47(11), P. 113502(1-24).

[9] Vorobiev A.M., Popov I.Y., Trifanova E.S. Resonance asymptotics for a pair quantum waveguides with common semitransparent perforated wall. Nanosystems: Physics, Chemistry, Mathematics, 2020, 11(6), P. 619-627.

[10] Kiselev A.A., Popov I.Yu. Indefinite metrics and scattering by a domain with small aperture. Mathematical Notes, 1995, 58(6), P. 1276-1285.

[11] Popov I.Yu. Helmholtz resonator and the operator extension theory in a space with an indefinite metrics. Matematicheskii sbornik, 1992, 183(3), P. 2-38; English translation in Russian Acad. Sci. Sb. Math., 1993, 75(2), P. 285-315.

[12] Shondin Yu.G. Quantum mechanical models in $\mathbb{R}_{n}$ connected with extensions of the energy operator in a Pontryagin space. Teoret. Mat. Fiz., 1988, 74, P. 331-344. English transl. in Theoret. and Math. Phys., $1988,74$.

[13] Faleeva M.P., Popov I.Y. On quantum bit coding by Gaussian beam modes for the quantum key distribution. Nanosystems: Physics, Chemistry, Mathematics, 2020, 11(6), P. 651-658.

[14] Vasylyev D.Yu., Semenov A.A., Vogel W. Atmospheric quantum channels with weak and strong turbulence. Phys. Rev. Lett., 2016, 117, P. 090501.

[15] Goncharenko A.M. Gaussian Beams of Light. Minsk, Science and Techn, 1977, 144 p. 